\title{
Device for Reduction Sparking of Slip Ring of Turbo Generator
}

\author{
Andrei N. Isakov, Andrei V. Andrusich, Igor E. Savraev \\ Pskov State University, Faculty of Electrical Engineering, Department of Drive and \\ automation systems. Address: 180760, Russia, Pskov, Lenin Square 2.
}

\begin{abstract}
Most modern power plants generating electric power equipped with synchronous generators. The correct operation of energy economy depends on reliability of such electrical machines.

In this article issue of increasing reliability of turbo generator by increasing reliability of its brush contact unit is considered. Description of brush contact unit and its possible damages is given. Equivalent electrical circuit of contact layer of brush contact unit is performed. The equations are formed and the dynamical simulation model consisting of electric source and two brush contact units is created. With help of this model alternative method of improving parameters of brush contact unit and increasing reliability of turbo generator at once is calculated and shown.

The implementation of the proposed method of increasing the turbo generator brush contact units reliability can have different technical performance, but the principle laid down in the method remains the same.
\end{abstract}

Keywords - contacts, brushes, turbogenerators, sparks, modeling.

\section{INTRODUCTION}

Reliable and uninterrupted power supply is placed at the center of the most important sites of the country, including the military and the civilian sector. Most modern power plants that provide electricity generation, equipped with synchronous machines [1]. The reliability of these machines depends on the work of almost all the energy complex.

According to the RAO UES of Russia, most failures, or $25.2 \%$ of all faults, turbo generators account for failure of brush-contact device (BCD) [2]. Stopping of turbo generator connected to its repair, can result in serious financial losses to the generating company. Therefore there is a need to improve the reliability of the unit $\mathrm{BCD}$ and turbo generators overall.

Brush-contact device of synchronous machine consists of a contact ring, located on the rotor, and several groups of brushes, brush holders located in the stator machine. Due to the pressure forces acting on the brush, the brush provided a mechanical contact with the ring, including at their relative movement.

The main part of the site BCD breakdowns associated with the failure of the brushes in connection with the processes occurring in the contact layer between the brush and the surface of the ring. Sliding between the brush and the surface of the ring arcing occurs, causing burn brush contact surface [3]. In addition to the contact layer derating brush sparking can cause a circular fire - emergency operation of $\mathrm{BCD}$, damaging turbo generator. Therefore, the main objective of increasing the service life of the unit will be reduced BCD sparking and reduce the possibility of a circular fire.

\section{MATERIALS AND METHODS}

From the point of view of an electrical circuit contact layer "brush-ring" is replaced by parallel connection of the resistance and capacitance [4], Fig. 1 .

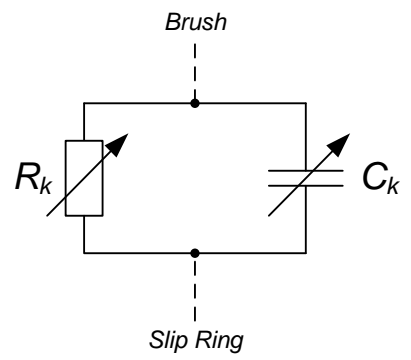

Fig. 1. The electrical equivalent circuit of the contact layer node moving current collection

Where $R_{k}, C_{k}$ - the resistance and capacitance of the contact layer "brush-ring."

It should be noted that the items included in the electrical equivalent circuit of the contact layer are variable parameters. This is due to the fact that the relative movement of the brushes and rings due to irregularities on the surface of the ring, the heterogeneity of the material brushes, micro vibrations brushes, brush holders and other factors change the parameters of the contact layer, which is reflected in its characteristics.

When feeding a closed loop consisting of a generator (electrical power source), two connecting conductors and the two nodes of brush-contact device electrical equivalent circuit of a closed circuit shown in Fig. 2. 


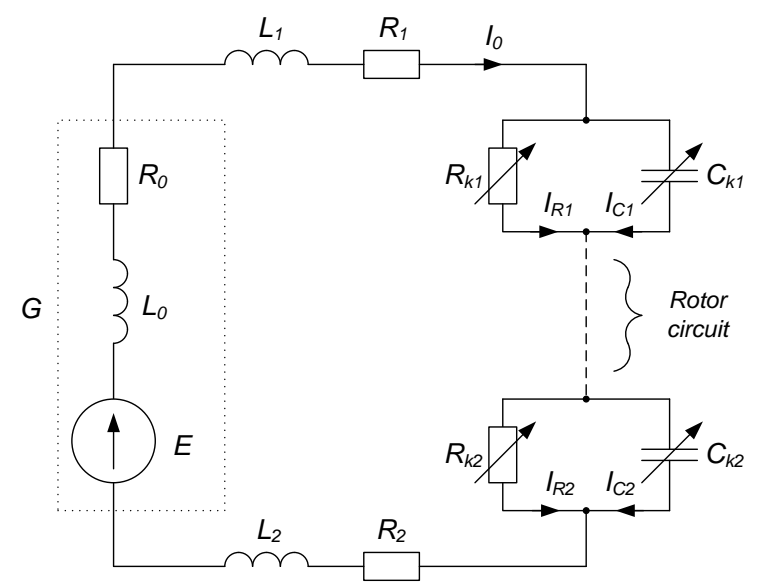

Fig. 2. The equivalent circuit of an electrical circuit with two nodes moving current collection

In Fig. 2 indicated:

$G$ - Generator circuit loop, which includes: $E$ electromotive force (EMF) generator, $L_{0}$ - selfinductance of the generator, $R_{0}$ - own resistance of the generator;

$L_{1}, R_{1}, L_{2}, R_{2}-$ the inductance and resistance of lead wires;

$R_{k 1}, C_{k 1}, R_{k 2}, C_{k 2}-$ the resistance and capacitance of the contact layer "brush-ring" for the respective groups of parallel sliding contacts.

Assuming that the rotor circuit is a bridge between the rings $\mathrm{BCD}$ and its resistance is zero, compiled by the laws of Kirchhoff's mathematical models for the equivalent circuit is as follows:
According to the obtained equations builds a dynamic simulation model in specific computer environment

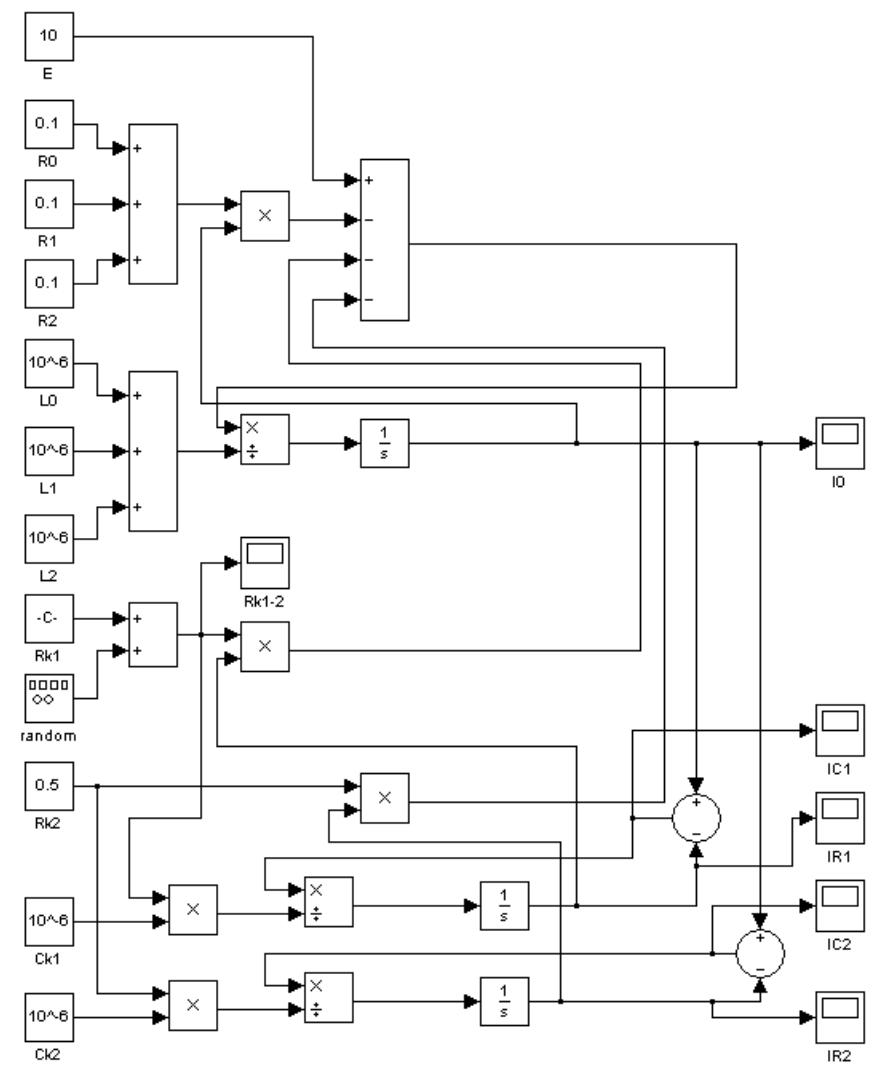

Fig. 3. Dynamic simulation model of current collection system moving in an specific computer environment

Built model system allows us to study the $I_{0} R_{0}+L_{0} \frac{d I_{0}}{d t}+L_{1} \frac{d I_{0}}{d t}+I_{0} R_{1}+I_{0} R_{k 1}+I_{0} R_{k 2}+I_{0} R_{2}+L_{2} \frac{d I_{0}}{d t}=E$ dependence of currents flowing through individual $d t d t \quad$ circuit elements, from changing their parameters.
Model the change in the current scheme for $I_{R 1} R_{k 1}-\frac{1}{C_{k 1}} \int I_{C 1} d t=0, \quad$ dynamically changing the resistance of the contact $\left\{I_{R 2} R_{k 2}-\frac{1}{C_{k 2}} \int I_{C 2} d t=0\right.$,

$I_{R 1}+I_{C 1}=I_{0}$, $I_{R 2}+I_{C 2}=I_{0}$.

With the help of the Laplace transform express currents of the above equations:
Initial data for the simulation:

$E=10 \mathrm{~V}$ - EMF generator;

$$
R_{0}=R_{1}=R_{2}=0,1 \mathrm{Ohm} \text { - resistance oscillator }
$$
circuit and connecting conductors;

$$
\begin{aligned}
& L_{0}=L_{1}=L_{2}=1 \mu H \text { - inductance of the generator } \\
& I_{0}=\frac{1}{p} \frac{1}{L_{0}+L_{1}+L_{2}}\left[E-\left(R_{0}+R_{1}+R_{2}\right) I_{0}-R_{k 1} I_{R 1}-R_{k 2} I_{R 2}\right], \quad \text { and connecting conductors; } \\
& \left\{\begin{array}{l}
I_{R 1}=\frac{1}{p} \frac{I_{C 1}}{C_{k 1} R_{k 1}}, \\
I_{R 2}=\frac{1}{p} \frac{I_{C 2}}{C_{k 2} R_{k 2}}, \\
I_{C 1}=I_{0}-I_{R 1},
\end{array}\right. \\
& \tilde{N}_{k 1}=C_{k 2}=1 \mu F \text { - capacity contact layers of two } \\
& \text { transitions "brush-ring"; } \\
& R_{k 2}=0,5 \mathrm{Ohm} \text { - resistance contact layer BCD second } \\
& R_{k 1}=0,01 . .500,01 \mathrm{Ohm}-\text { resistance of the contact } \\
& \text { assembly BCD. }
\end{aligned}
$$

It should be noted that the data presented resistance contact layer of the first node BCD varies within these ranges at random. [5] 
The result of the model are the following timing diagrams of the currents in the main chain (Fig. 4) and the current (Fig. 5):

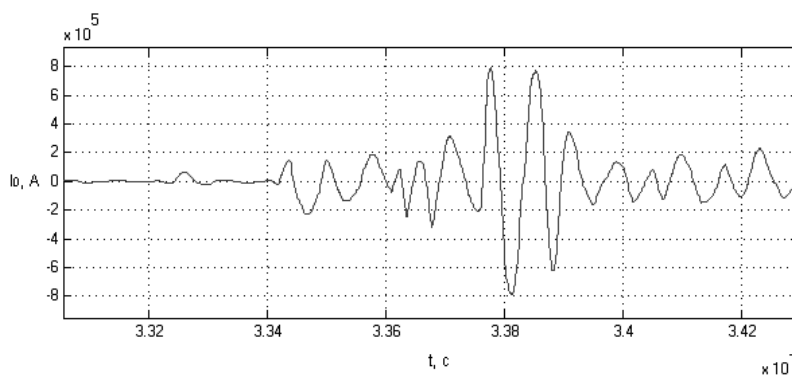

Fig. 4. The timing diagram of the main current in a circuit with two nodes moving current collection

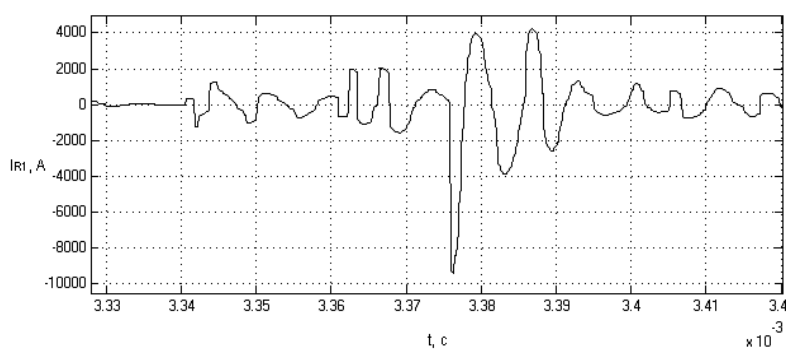

Fig. 5. The timing diagram of current through the resistance of the contact layer of the first node BCD

Seen from the figures that the dynamic change of the resistance of the contact layer having short but very significant emissions of the electric current flowing in the circuit, which can reach values of several thousand amperes, with an average current in amperes. This is explained by the fact that dynamically changes the resistance of the contact layer of the first node $\mathrm{BCD}$ provokes parametric resonance in the system, causing a sharp increase in the amplitude of the currents in the circuit.

To combat the parametric resonance arising in the circuit with two nodes $\mathrm{BCD}$, increase the capacity of the contact layer of the first node to the value $\tilde{N}_{k 1}=1 \mathrm{mF}$ BCD. The result of the model in this case (Fig. 6 and Fig. 7):

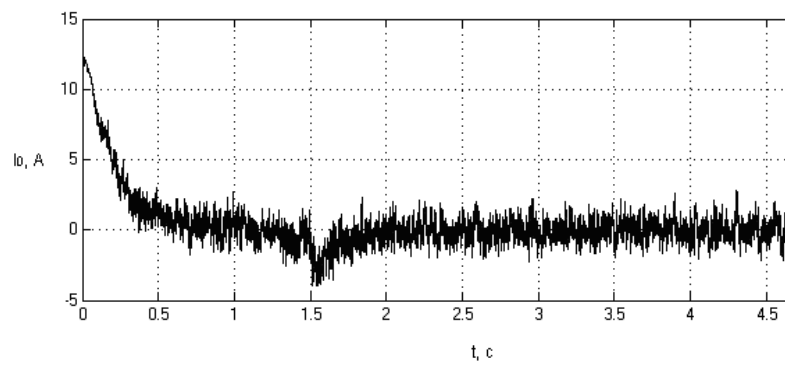

Fig. 6. The timing diagram of the main current in a circuit with two nodes moving current collection

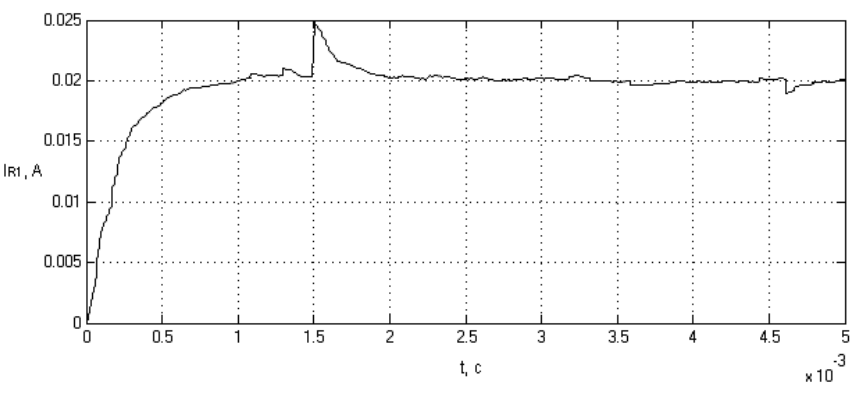

Fig. 7. The timing diagram of current through the resistance of the contact layer of the first node BCD

\section{RESULTS AND DISCUSSION}

Seen from the figures that when the capacity of the contact layer dynamic currents in the circuit have decreased significantly, from a few thousand to a few Amperes with RMS value about few Amperes. This result shows that an artificial increase in the capacity of transition "brush-ring" leads to a reduction of emissions of current flowing through the junction, causing a reduction in sparking host BCD and decrease the possibility of a circular fire.

Thus, increasing the capacity of transition "brushring" leads to a drastic reduction in dynamic currents affecting the work BCD. Decrease in the amplitude of dynamic currents will reduce sparking BCD in the contact layer, which would prolong the operational life of the brushes, and reduce the risk of circular fire. This in turn increases the overall reliability of the turbo generators.

The implementation of this approach to increase the reliability of nodes BCD can have different technical performance, but the principle laid down in the approach remains the same.

\section{ACKNOWLEDGMENTS}

This study was supported by Pskov State University.

\section{$\mathrm{V}$ REFERENCES}

[1] I.P. Kopylov. Electrical Machines. - Moscow: Logos, 2000. $607 \mathrm{p}$.

[2] ELISA, "Problems of BCD". [Online]. Available: http://npfelisa.ru/problemy-shka

[3] S.I. Kachin, O.S. Kachin. "Modeling of processes of wear electric brushes universal motors considering mechanical factors" / / Electricity. - 2009. - № 12. - pp. 68-70.

[4] I.V. Plokhov. "Comprehensive diagnosis and prediction of technical state of nodes moving current collection turbo generators" / Author. Dissertation. on competition. step. Doctor. tech. Science. - Saint-Petersburg: SPbGTU, 2001. $36 \mathrm{p}$.

[5] Probability and Statistics: Encyclopedia / Ch. Ed. Prokhorov Y.V. - Moscow.: the Great Russian Encyclopedia, 1999. - 910 p. 